\title{
FINITE TIME STABILIZATION OF NONLINEAR OSCILLATORS SUBJECT TO DRY FRICTION
}

\author{
Samir Adly \\ Laboratoire LACO, Université de Limoges, 123 avenue Albert Thomas, 87060 Limoges \\ Cedex, France \\ samir.adly@unilim.fr
}

Hedy Attouch

Laboratoire ACSIOM, Université Montpellier II, Place Eugène Bataillon, 34095 Montpellier Cedex 05, France

attouch@math.univ-montp2.fr

\author{
Alexandre Cabot \\ Laboratoire LACO, Université de Limoges, 123 avenue Albert Thomas, 87060 Limoges \\ Cedex, France \\ alexandre.cabot@unilim.fr
}

Abstract Given a smooth function $f: \mathbb{R}^{n} \rightarrow \mathbb{R}$ and a convex function $\Phi: \mathbb{R}^{n} \rightarrow \mathbb{R}$, we consider the following differential inclusion:

$(S) \quad \ddot{x}(t)+\partial \Phi(\dot{x}(t))+\nabla f(x(t)) \ni 0, \quad t \geq 0$,

where $\partial \Phi$ denotes the subdifferential of $\Phi$. The term $\partial \Phi(\dot{x})$ is strongly related with the notion of friction in unilateral mechanics. The trajectories of $(S)$ are shown to converge toward a stationary solution of $(S)$. Under the additional assumption that $0 \in \operatorname{int} \partial \Phi(0)$ (case of a dry friction), we prove that the limit is achieved in a finite time. This result may have interesting consequences in optimization.

Keywords: Dissipative dynamical system, differential inclusion, dry friction, finite time convergence. 


\section{Introduction and notation}

Throughout the paper, $H=\mathbb{R}^{n}$ is equipped with the euclidean scalar product $\langle.,$.$\rangle and the corresponding norm |$.$| . Let f: \mathbb{R}^{n} \rightarrow \mathbb{R}$ a function of class $\mathcal{C}^{1}$ that we wish to minimize over $\mathbb{R}^{n}$. A powerful method consists in following the trajectories of a gradient-based dynamical system. If the dynamics is dissipative, the trajectories will hopefully converge toward a critical point of the potential $f$. In the past ten years, much attention has been brought to the study of second-order in time dynamical systems. Because of the inertial aspects, the dynamics of such systems do not stop at each minimum point. These methods are endowed with better exploration properties than first-order ones. Among them, let us quote the "Heavy Ball with Friction" system:

$(H B F)$

$$
\ddot{x}(t)+\gamma \dot{x}(t)+\nabla f(x(t))=0, \quad t \geq 0, \quad \gamma>0,
$$

which has been initiated by Polyak (Polyack, 1964). Several authors have recently studied the properties of the $(H B F)$ system ( $c f$. Alvarez (Alvarez, 2000), Attouch-Goudou-Redont (Attouch et al., 2000)). The main numerical drawback of the $(H B F)$ method comes from the oscillatory behaviour of the trajectory near the minimum. A first way to improve the $(H B F)$ method consists in introducing a second-order information on $f$ via the hessian matrix. In mechanical terms, this amounts to replacing the viscous friction $-\gamma \dot{x}$ by a hessian-driven one (and eventually combining both frictions). This approach has been studied by AttouchRedont (Attouch and Redont, 2001) and Alvarez-Attouch-Bolte-Redont (Alvarez et al., 2002). Our point of view in this paper is quite different and consists in strengthening the friction when the velocity $\dot{x}$ vanishes. This approach is inspired by mechanical models involving dry friction. A very wide literature is devoted to the contact problems with Coulomb friction (see for example (Amassad and Fabre, 2003, Cadivel et al., 2000, Dumont et al., 2000, Eck and Jarusek, 2000, Han and Sofonea, 2002, Jean, 1988)). Let us recall that, in the one dimensional setting, the classical Coulomb friction is given by $-\alpha \operatorname{Sgn}(\dot{x})$, where $\alpha>0$ and $\operatorname{Sgn}$ is the set-valued sign function: $\operatorname{Sgn}(x)=1$ if $x>0$, $\operatorname{Sgn}(x)=-1$ if $x<0$ and $\operatorname{Sgn}(0)=[-1,1]$. The multivalued function Sgn coincides with the subdifferential of the function $\mathbb{R} \ni x \rightarrow|x|$. These considerations lead us to study the following differential inclusion:

$$
\ddot{x}(t)+\partial \Phi(\dot{x}(t))+\nabla f(x(t)) \ni 0, \quad t \geq 0,
$$

where $\partial \Phi$ denotes the subdifferential of the convex function $\Phi: \mathbb{R}^{n} \rightarrow$ $\mathbb{R}$. Notice that in unilateral mechanics, the modelling by differential inclusions has been initiated by Moreau (Moreau, 1977, Moreau, 1985) 
and has been intensively studied by many authors (Mabrouk, Monteiro Marques, 1993, Schatzman, 1978), to quote only some of them. The formulation $(S)$ allows to recover a large variety of friction models. For example, the function $\Phi=\alpha|$.$| corresponds to the Coulomb friction$ and on the other hand, $\Phi=\gamma|\cdot|^{2} / 2$ gives the viscous friction and the associated $(H B F)$ system. Notice also that the function $\Phi=\beta|\cdot|^{p} / p$ $(p \in] 1,2[)$ generates an intermediate situation, which has been studied by Amann-Díaz (Amann and Díaz, 2003) and Díaz-Liñán (Díaz and Liñán, 2001) under the terminology of "strong friction".

The system $(S)$ defined above may have interesting applications in cognitive sciences and decision sciences (mathematical economy, game theory,...). Indeed, the friction corresponds in this case to the cost of changing (cost of leaving a routine, cost of exploration by tests and errors, cost of dissimilarity,...). The viscous friction is associated to a quadratic cost with respect to the distance to the rest position. On the other hand, the dry friction corresponds to a linear cost so that the changes are relatively expensive. This last model may represent the inertial aspects of human behaviours. The introduction of costs of changing is an important characteristic of the theory of bounded rationality, $c f$. (Conlisk, 1996, Rubinstein, 1998, Sobel, 2000).

For any couple of initial conditions, the inclusion $(S)$ is shown to admit a unique solution $x:\left[0,+\infty\left[\rightarrow \mathbb{R}^{n}\right.\right.$ of class $\mathcal{C}^{1}$ satisfying $(S)$ almost everywhere. The behaviour at infinity of the $(S)$ trajectory essentially depends on the nature of the friction term $\Phi$ near the origin. When $\partial \Phi(0)=\{0\}$, the gradient $\nabla f(x(t))$ tends to 0 when $t$ tends to $+\infty$; however the convergence of the trajectories may not hold without further assumptions on $f$ (like convexity or analyticity). On the other hand, when the friction is dry, i.e. $0 \in \operatorname{int} \partial \Phi(0)$ the convergence of the trajectories automatically holds. The main result of the paper consists in showing that the limit is achieved in a finite time (see Theorem 24.8). Such a limit $x^{*}$ is a solution of the inclusion: $-\nabla f\left(x^{*}\right) \in \partial \Phi(0)$, i.e. $x^{*}$ is a stationary solution of $(S)$. Since our goal is to minimize $f$, we must choose a function $\Phi$ having a "small" subdifferential set $\partial \Phi(0)$, so as to force the trajectories to converge close to the "exact" critical points. In the various contexts recalled above (mechanics, cognitive sciences,...), the finite time stabilization of the system is meaningful. Such a result in finite dimension opens interesting perspectives concerning similar results for mechanical and physical systems with infinite degrees of freedom.

The paper is organized as follows. In section 2, we state the existence and uniqueness of the solution satisfying the Cauchy problem associated with $(S)$. For the sake of readability, the proof of this result is postponed to section 4 . The technique consists in using the Moreau-Yosida approx- 
imates of $\Phi$, then establishing uniform estimates and finally passing to the limit by means of compacity arguments. Section 3 is devoted to the asymptotic study of $(S)$ in the case $0 \in \operatorname{int} \partial \Phi(0)$.

\section{The second order differential inclusion $(S)$}

\subsection{Mechanical example}

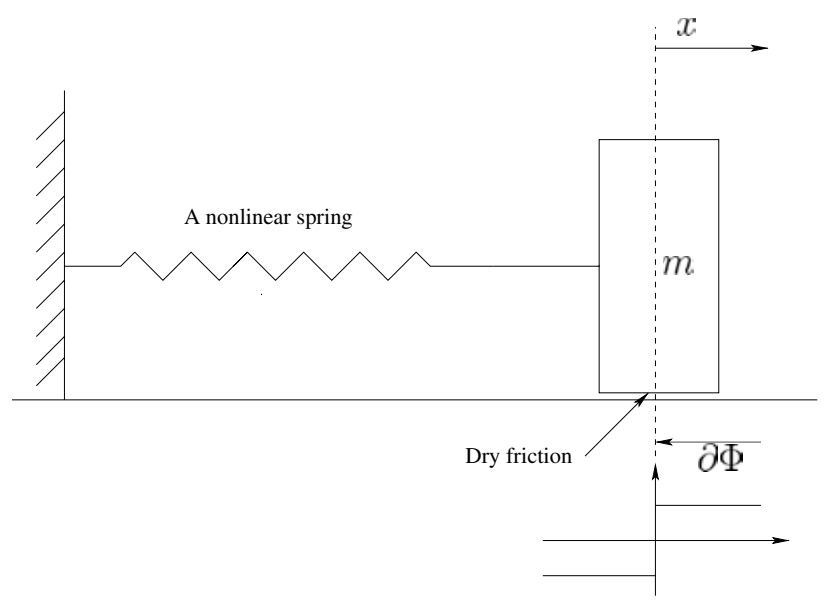

Figure 24.1. A nonlinear oscillator subject to dry friction.

Let us consider the nonlinear oscillator system whose dynamics equation is given by:

$$
m \ddot{x}+g(x) \in-\partial \Phi(\dot{x}),
$$

Equation (24.1) describes the motion of a mass $m$ sliding on a surface and attached to a nonlinear spring. The term $-\partial \Phi(\dot{x})$ represents the dry frictional contact of the mass on the surface and $-g(x)$ corresponds to the force exerted by the spring on the mass. In large displacement operation springs are nonlinear: this is the case for example of the wool felt used for piano hammers which acts as a nonlinear hardening spring. Experimental measurements show that the nonlinear relationship between applied force and felt compression is of the form: $g(x)=k x^{p} \quad(x \geq 0)$, where $k$ is a generalized stiffness coefficient and $p$ is called the effective nonlinearity exponent. Static measurements typically produce values of $p$ in the interval $[2.2,3.5]$. For more details about the hardness of a piano hammer, we refer to (Russell, 1997). In general, for a nonlinear spring, the stiffness can be broken up into two parts: $g(x)=k_{0} x+k_{1}|x|^{p} \operatorname{sgn}(x)$, where $k_{0}$ and $k_{1}$ are the constants for the linear and nonlinear parts re- 
spectively. Here sgn denotes the classical sign function. The function $g$ derives from the potential $f$ given by:

$$
f(x)=\frac{k_{0}}{2}|x|^{2}+\frac{k_{1}}{p+1}|x|^{p+1} .
$$

The dry friction is usually modelled by the Coulomb one: $\Phi_{0}(\dot{x})=$ $\gamma_{0}|\dot{x}|$, where $\gamma_{0}$ is a positive coefficient. The friction force is then given by $-\partial \Phi_{0}(\dot{x})=-\gamma_{0} \operatorname{Sgn}(\dot{x})$ where Sgn is the multivalued operator defined by: $\operatorname{Sgn}(\dot{x})=1$ if $\dot{x}>0, \operatorname{Sgn}(\dot{x})=-1$ if $\dot{x}<0$ and $\operatorname{Sgn}(0)=[-1,1]$. In many situations, the dry friction is combined with a viscous friction $-\gamma_{1} \dot{x}$, so that the total friction term equals:

$$
\Phi(\dot{x})=\gamma_{0}|\dot{x}|+\frac{\gamma_{1}}{2}|\dot{x}|^{2} .
$$

For other examples of nonlinear oscillators involving dry friction, we refer the reader to (Adly and Goeleven, 2004, Cadivel et al., 2000, Dumont et al., 2000).

\subsection{Global existence and uniqueness result}

Consider two functions $\Phi: \mathbb{R}^{n} \rightarrow \mathbb{R}$ and $f: \mathbb{R}^{n} \rightarrow \mathbb{R}$ satisfying respectively the following assumptions:

$\left(\mathcal{H}_{\Phi}-i\right) \Phi$ is convex; $\left(\mathcal{H}_{\Phi}-i i\right) \min _{x \in \mathbb{R}^{n}} \Phi(x)=\Phi(0)=0$.

$\left(\mathcal{H}_{f}-i\right) f$ is of class $\mathcal{C}^{1}$ and $\nabla f$ is Lipschitz continuous on the bounded subsets of $\mathbb{R}^{n}$. $\left(\mathcal{H}_{f}-i i\right) f$ is bounded from below.

Let us consider the problem of finding a continuous function $x$ such that $\dot{x} \in L_{l o c}^{\infty}\left(\left[0,+\infty\left[, \mathbb{R}^{n}\right)\right.\right.$ and satisfying

$$
\ddot{x}(t)+\partial \Phi(\dot{x}(t))+\nabla f(x(t)) \ni 0, \quad t \geq 0 .
$$

We start with a general result of existence and uniqueness for the associated Cauchy problem.

Theorem 24.1 (Existence And Uniqueness) Assume that hypotheses $\left(\mathcal{H}_{f}-i, i i\right)$ and $\left(\mathcal{H}_{\Phi}-i, i i\right)$ hold. Then, for every $\left(x_{0}, \dot{x}_{0}\right) \in \mathbb{R}^{n} \times \mathbb{R}^{n}$, there exists a unique solution $x:\left[0,+\infty\left[\rightarrow \mathbb{R}^{n}\right.\right.$ of $(S)$ in the following sense:

(a) $x \in \mathcal{C}^{1}\left(\left[0,+\infty\left[, \mathbb{R}^{n}\right)\right.\right.$ and $x \in \mathcal{W}^{2,+\infty}\left([0, T], \mathbb{R}^{n}\right)$ for every $T>0$.

(b) $(S)$ is satisfied for almost every $t \in[0,+\infty[$.

(c) $x(0)=x_{0}$ and $\dot{x}(0)=\dot{x}_{0}$. 
The proof of the uniqueness in Theorem 24.1 is classical and left to the reader. The problem of the existence is postponed to section 4 .

REMARK 24.2 We note that the second-order system $(S)$ can be written as a first-order one in $\mathbb{R}^{n} \times \mathbb{R}^{n}$ :

$$
\left(S^{*}\right) \quad \dot{X}+\partial \varphi(X)+F(X) \ni 0,
$$

with $X(t)=(x(t), \dot{x}(t))=\left(x_{1}, x_{2}\right), \varphi\left(x_{1}, x_{2}\right)=\Phi\left(x_{2}\right)$ and $F\left(x_{1}, x_{2}\right)=$ $\left(-x_{2}, \nabla f\left(x_{1}\right)\right)$. The system $\left(S^{*}\right)$ can be seen as a first-order dynamical system governed by a perturbation of a maximal monotone operator $\partial \varphi$ with a locally Lipschitz operator $F$. We can not apply general existence and uniqueness results like Kato's Theorem (see Brezis (Brézis, 1972), Kato (Kato, 1970)) here since the perturbation function $F$ is not Lipschitz continuous on the whole space $\mathbb{R}^{n} \times \mathbb{R}^{n}$.

\section{Finite time convergence under dry friction \\ 3.1 First asymptotical results}

Once the global existence and uniqueness is acquired in the study of $(S)$, we wish to investigate the asymptotical properties of $(S)$. The key tool is the existence of a Lyapounov function $E$ emanating from the mechanical interpretation of $(S)$.

Proposition 24.3 Under the assumptions $\left(\mathcal{H}_{f}-i, i i\right)$ and $\left(\mathcal{H}_{\Phi}-i, i i\right)$, consider the unique solution of the $(S)$ system and define the energy function by $E(t):=\frac{1}{2}|\dot{x}(t)|^{2}+f(x(t))$. Then, the following holds:

(i) For almost every $t \in \mathbb{R}_{+}$,

$$
\dot{E}(t) \leq-\Phi(\dot{x}(t)) \leq 0,
$$

and hence $E$ is a Lyapounov function for the $(S)$ system.

(ii) $\dot{x} \in L^{\infty}\left(\left[0,+\infty\left[, \mathbb{R}^{n}\right)\right.\right.$ and $\Phi(\dot{x}) \in L^{1}([0,+\infty[, \mathbb{R})$.

Proof. (i) Let $D$ be the subset of $\mathbb{R}_{+}$on which the map $\dot{x}$ is derivable and the inclusion $(S)$ is satisfied. Since the function $\dot{x}$ is absolutely continuous and taking into account Theorem 24.1 (b), it is clear that the set $\mathbb{R}_{+} \backslash D$ is negligible. Let us prove that inequality (24.2) is satisfied for every $t \in D$. By differentiating the expression of $E$, we find:

$$
\begin{aligned}
\forall t \in D, \quad \dot{E}(t) & =\langle\ddot{x}(t), \dot{x}(t)\rangle+\langle\nabla f(x(t)), \dot{x}(t)\rangle \\
& =-\langle-\ddot{x}(t)-\nabla f(x(t)), \dot{x}(t)\rangle .
\end{aligned}
$$

From the fact that $-\ddot{x}(t)-\nabla f(x(t)) \in \partial \Phi(\dot{x}(t))$, we deduce that

$$
\dot{E}(t) \leq-(\Phi(\dot{x}(t))-\Phi(0))=-\Phi(\dot{x}(t))
$$


and hence formula (24.2) holds for every $t \in D$.

(ii) In view of (i), the function $E$ is non increasing and hence

$$
E(t)=\frac{1}{2}|\dot{x}(t)|^{2}+f(x(t)) \leq E(0) .
$$

We then deduce that $\frac{1}{2}|\dot{x}(t)|^{2} \leq E(0)-\inf f$, i.e. $\dot{x} \in L^{\infty}\left(\left[0,+\infty\left[, \mathbb{R}^{n}\right)\right.\right.$. Now integrate inequality (24.2) between 0 and $t$; we obtain

$$
\int_{0}^{t} \Phi(\dot{x}(s)) d s \leq E(0)-E(t) \leq E(0)-\inf f .
$$

Taking the limit as $t \rightarrow+\infty$, we obtain $\Phi(\dot{x}) \in L^{1}([0,+\infty[, \mathbb{R})$.

From now on, we will assume that the term $\Phi$ strictly dissipates the energy when the velocity $|\dot{x}|$ is positive, which amounts to suppose that: $\left(\mathcal{H}_{\Phi}-i i i\right) \operatorname{argmin} \Phi=\{0\}$.

An essential step in the asymptotical study of $(S)$ consists in proving that the velocity $\dot{x}$ tends to 0 when $t \rightarrow+\infty$.

Proposition 24.4 Under hypotheses $\left(\mathcal{H}_{f}-i, i i\right)$ and $\left(\mathcal{H}_{\Phi}-i, i i, i i i\right)$, let $x$ be the unique solution of the $(S)$ system. If $x \in L^{\infty}\left(\left[0,+\infty\left[, \mathbb{R}^{n}\right)\right.\right.$, then we have $\lim _{t \rightarrow+\infty} \dot{x}(t)=0$.

Proof. Since $\nabla f$ is Lipschitz continuous on the bounded sets, it is clear that

$$
t \mapsto \nabla f(x(t)) \quad \text { is bounded. }
$$

From Proposition 24.3 (ii), the map $\dot{x}$ is bounded. In view of the boundedness of $\partial \Phi$ on bounded sets, this implies the existence of a bounded set $B \subset \mathbb{R}^{n}$ such that for every $t \in \mathbb{R}_{+}$,

$$
\partial \Phi(\dot{x}(t)) \subset B .
$$

As a consequence, we deduce from (24.3), (24.4) and $(S)$ that $\ddot{x} \in$ $L^{\infty}\left(\left[0,+\infty\left[, \mathbb{R}^{n}\right)\right.\right.$, i.e. $\dot{x} \in \operatorname{Lip}\left(\left[0,+\infty\left[, \mathbb{R}^{n}\right)\right.\right.$. Since the function $\Phi$ is Lipschitz continuous on the bounded sets, it is clear that $\Phi(\dot{x}) \in \operatorname{Lip}([0,+\infty[, \mathbb{R})$. This combined with the fact that $\Phi(\dot{x}) \in L^{1}([0,+\infty[, \mathbb{R})$ classically implies that $\lim _{t \rightarrow+\infty} \Phi(\dot{x})(t)=0$.

Let $\bar{u} \in \mathbb{R}^{n}$ be a cluster point of the bounded set $\{\dot{x}(t), t \geq 0\}$. There exists a sequence $\left(t_{n}\right)$ such that $\lim _{n \rightarrow+\infty} \dot{x}\left(t_{n}\right)=\bar{u}$. From the continuity of $\Phi$, we infer that $\Phi(\bar{u})=0$, i.e. $\bar{u} \in \operatorname{argmin} \Phi=\{0\}$. Consequently, 0 is the unique limit point of $\{\dot{x}(t), t \geq 0\}$ and therefore $\lim _{t \rightarrow+\infty} \dot{x}(t)=0$.

To go further in the asymptotical results relative to $(S)$, we have to specify our assumptions on the function $\Phi$. Indeed, the behaviour of 
$x$ at infinity depends on the nature of the friction near the origin. A "strong" friction can force the trajectories of $(S)$ to converge fastly and even in a finite time. These situations of "strong" friction are analyzed in the next section, where the adequate condition on $\Phi$ is shown to be $0 \in \operatorname{int} \partial \Phi(0)$.

\subsection{Case of the dry friction: $0 \in \operatorname{int} \partial \Phi(0)$}

Let us first remark that the condition $0 \in \operatorname{int} \partial \Phi(0)$ amounts to saying that the function $\Phi$ is minorized by $\alpha \mid$. |, for some $\alpha>0$. Indeed, we have:

Lemma 24.5 Let $\Phi: \mathbb{R}^{n} \rightarrow \mathbb{R}$ a convex function satisfying $\Phi(0)=0$. Then the following assertions are equivalent:

(i) $\Phi \geq \alpha|$.$| , for some \alpha>0$.

(ii) $0 \in \operatorname{int} \partial \Phi(0)$.

The proof of Lemma 24.5 is elementary and left to the reader. If $0 \in$ int $\partial \Phi(0)$, it is clear, in view of the previous lemma that assumption $\left(\mathcal{H}_{\Phi}-i i i\right)$ is automatically satisfied. The following result shows the convergence of the $(S)$ trajectories under the condition $0 \in$ int $\partial \Phi(0)$.

Theorem 24.6 Under the hypotheses $\left(\mathcal{H}_{\Phi}-i, i i\right)$ and $\left(\mathcal{H}_{f}-i, i i\right)$, assume moreover that $0 \in$ int $\partial \Phi(0)$. Then the unique solution $x$ of $(S)$ satisfies the following assertions:

(i) $|\dot{x}| \in L^{1}\left(\left[0,+\infty[, \mathbb{R})\right.\right.$ and therefore $x_{\infty}:=\lim _{t \rightarrow+\infty} x(t)$ exists.

(ii) The limit point $x_{\infty}$ verifies

$$
-\nabla f\left(x_{\infty}\right) \in \partial \Phi(0)
$$

i.e. $x_{\infty}$ is an equilibrium point of $(S)$.

Proof. (i) From Lemma 24.5, the condition $0 \in \operatorname{int} \partial \Phi(0)$ implies the existence of $\alpha>0$ such that $\Phi \geq \alpha|$.$| . On the other hand, from$ Proposition 24.3 (ii), we have $\Phi(\dot{x}) \in L^{1}([0,+\infty[, \mathbb{R})$ and it follows that $|\dot{x}| \in L^{1}([0,+\infty[, \mathbb{R})$. The convergence of the trajectory $x($.$) immediately$ results from the equality $x(t)=x(0)+\int_{0}^{t} \dot{x}(s) d s$.

(ii) Let us argue by contradiction and assume that the set $C:=$ $\partial \Phi(0)+\nabla f\left(x_{\infty}\right)$ does not contain 0 . It is then possible to strictly separate the convex compact set $\{0\}$ from the closed convex set $C$. More precisely, there exist $p \in \mathbb{R}^{n}$ and $m \in \mathbb{R}_{+}^{*}$ such that, for every $x \in C$, $\langle x, p\rangle>m$, which amounts to say that $C$ is contained in the open halfspace $\mathcal{H}_{p, m}$ defined by

$$
\mathcal{H}_{p, m}:=\left\{x \in \mathbb{R}^{n}, \quad\langle x, p\rangle>m\right\} .
$$


Let us prove that for $t$ large enough, we have

$$
\partial \Phi(\dot{x}(t))+\nabla f(x(t)) \subset \mathcal{H}_{p, m} .
$$

If this was not true, there would exist a sequence $\left(t_{n}\right)$ tending to $+\infty$ such that $\partial \Phi\left(\dot{x}\left(t_{n}\right)\right)+\nabla f\left(x\left(t_{n}\right)\right) \not \subset \mathcal{H}_{p, m}$. This means that, for every $n \in \mathbb{N}$, there exists $u_{n} \in \partial \Phi\left(\dot{x}\left(t_{n}\right)\right)$ such that

$$
\left\langle u_{n}+\nabla f\left(x\left(t_{n}\right)\right), p\right\rangle \leq m .
$$

Since the operator $\partial \Phi$ is bounded on the bounded sets, the sequence $\left(u_{n}\right)$ is bounded. Therefore, there exists $\bar{u} \in \mathbb{R}^{n}$ and a converging subsequence of $\left(u_{n}\right)$, still denoted by $\left(u_{n}\right)$ such that $\lim _{n \rightarrow+\infty} u_{n}=\bar{u}$. On the other hand, we have $\lim _{n \rightarrow+\infty} \dot{x}\left(t_{n}\right)=0$ and using the graph-closedness property of the operator $\partial \Phi$ in $\mathbb{R}^{n} \times \mathbb{R}^{n}$, we conclude that $\bar{u} \in \partial \Phi(0)$. Let us now take the limit in (24.7) when $n \rightarrow+\infty$ :

$$
\left\langle\bar{u}+\nabla f\left(x_{\infty}\right), p\right\rangle \leq m,
$$

i.e. $\bar{u}+\nabla f\left(x_{\infty}\right) \notin \mathcal{H}_{p, m}$. Since $\bar{u} \in \partial \Phi(0)$, we deduce that $C=\partial \Phi(0)+$ $\nabla f\left(x_{\infty}\right) \not \subset \mathcal{H}_{p, m}$, a contradiction. Hence, we have proved that there exists $t_{0} \geq 0$ such that the inclusion (24.6) holds for $t \geq t_{0}$. Coming back to the $(S)$ system, we infer that, for almost every $t \geq t_{0},\langle-\ddot{x}(t), p\rangle>m$. Integrating this inequality between $t_{0}$ and $t$ immediately yields

$$
\langle-\dot{x}(t), p\rangle>\left\langle-\dot{x}\left(t_{0}\right), p\right\rangle+m\left(t-t_{0}\right)
$$

and we deduce that $\lim _{t \rightarrow+\infty}\langle\dot{x}(t), p\rangle=-\infty$, a contradiction with the fact that $\dot{x} \in L^{\infty}\left(\left[0,+\infty\left[, \mathbb{R}^{n}\right)\right.\right.$. As a consequence, the initial assumption $0 \notin \partial \Phi(0)+\nabla f\left(x_{\infty}\right)$ is false, which ends the proof.

REMARK 24.7 We notice that in the case of a dry friction, the proof of the convergence of the trajectories $x($.$) in Theorem 24.6$ is elementary. This contrasts with situations involving viscous friction, where the velocity $\dot{x}$ is in $L^{2}$ but not in $L^{1}$ in general. In the case of a linear damped dynamics, convergence may not hold without further assumptions on $f$ like convexity or analyticity (see for example (Alvarez, 2000, Attouch et al., 2000)). However the convergence of the $(S)$ trajectories under dry friction has a counterpart: in view of (24.5), the limit is just an "approximate" critical point of $f$. Since our goal is to minimize the function $f$, we will have to choose a function $\Phi$ whose subdifferential set $\partial \Phi(0)$ is "relatively small".

The most remarkable property of the dry friction is the convergence in finite time of the $(S)$ trajectories. The following statement is quite general and there are no further assumptions on the potential $f$. 
Theorem 24.8 (Finite time CONVERGEnCE) Under the hypotheses of Theorem 24.6, let $x$ be the unique solution of $(S)$ and let $x_{\infty} \in \mathbb{R}^{n}$ be defined by $x_{\infty}:=\lim _{t \rightarrow+\infty} x(t)$. If $-\nabla f\left(x_{\infty}\right) \notin \mathrm{bd}(\partial \Phi(0))$, then there exists $t_{1} \geq 0$ such that $x(t)=x_{\infty}$ for every $t \geq t_{1}$.

Proof. From Theorem 24.6, the limit point $x_{\infty}$ necessarily fulfils $-\nabla f\left(x_{\infty}\right) \in$ $\partial \Phi(0)$, so that the assumption $-\nabla f\left(x_{\infty}\right) \notin \mathrm{bd}(\partial \Phi(0))$ is equivalent to $-\nabla f\left(x_{\infty}\right) \in \operatorname{int}(\partial \Phi(0))$. This implies the existence of $\varepsilon>0$ such that

$$
-\nabla f\left(x_{\infty}\right)+\mathbb{B}(0,2 \varepsilon) \subset \partial \Phi(0) .
$$

On the other hand, since $\lim _{t \rightarrow+\infty} \nabla f(x(t))=\nabla f\left(x_{\infty}\right)$, there exists $t_{0} \geq 0$ such that for every $t \geq t_{0}$, we have

$$
\nabla f(x(t)) \in \nabla f\left(x_{\infty}\right)+\mathbb{B}(0, \varepsilon) .
$$

Hence,

$$
-\nabla f(x(t))+\mathbb{B}(0, \varepsilon) \subset-\nabla f\left(x_{\infty}\right)+\mathbb{B}(0,2 \varepsilon) \subset \partial \Phi(0) .
$$

This means that, for every $t \geq t_{0}$ and for every $u \in \mathbb{B}(0,1)$, we have:

$$
-\nabla f(x(t))+\varepsilon u \in \partial \Phi(0) .
$$

Thus, for every $t \geq t_{0}$, we deduce

$$
\forall u \in \mathbb{B}(0,1), \quad \Phi(\dot{x}(t)) \geq\langle-\nabla f(x(t))+\varepsilon u, \dot{x}(t)\rangle .
$$

Taking the supremum over $u \in \mathbb{B}(0,1)$, we obtain for every $t \geq t_{0}$,

$$
\Phi(\dot{x}(t)) \geq\langle-\nabla f(x(t)), \dot{x}(t)\rangle+\varepsilon|\dot{x}(t)| .
$$

On the other hand, the inequality (24.2) of energy decay can be rewritten as:

$$
\frac{1}{2} \frac{d}{d t}|\dot{x}(t)|^{2}+\langle\nabla f(x(t)), \dot{x}(t)\rangle+\Phi(\dot{x}(t)) \leq 0 \quad \text { a.e. on }[0,+\infty[\text {. }
$$

By combining (24.8) and (24.9), we get

$$
\frac{1}{2} \frac{d}{d t}|\dot{x}(t)|^{2}+\varepsilon|\dot{x}(t)| \leq 0 .
$$

By setting $h(t):=|\dot{x}(t)|^{2}$, it is clear that relation (24.10) can be rewritten as the following differential inequality:

$$
\dot{h}(t)+2 \varepsilon \sqrt{h(t)} \leq 0 \quad \text { a.e. on }[0,+\infty[.
$$


Let us prove that there exists $t_{1} \geq t_{0}$ such that $h\left(t_{1}\right)=0$. Assume, on the contrary that for every $t \geq t_{0}, h(t)>0$. Dividing (24.11) by $\sqrt{h(t)}$ and integrating on $\left[t_{0}, t\right]$, we obtain

$$
\sqrt{h(t)}-\sqrt{h\left(t_{0}\right)} \leq-\varepsilon\left(t-t_{0}\right) .
$$

Letting $t \rightarrow+\infty$, the previous inequality leads to $\lim _{t \rightarrow+\infty} \sqrt{h(t)}=$ $-\infty$, a contradiction, whence the existence of $t_{1} \geq t_{0}$ satisfying $h\left(t_{1}\right)=0$.

From (24.11), we deduce that $\dot{h}(t) \leq 0$ almost everywhere and hence $h(t) \leq h\left(t_{1}\right)=0$, for every $t \geq t_{1}$. We conclude that $|\dot{x}(t)|=0$ for every $t \in\left[t_{1},+\infty\left[\right.\right.$, i.e. $x(t)=x_{\infty}$ for every $t \in\left[t_{1},+\infty[\right.$.

We may notice that the conclusions of Theorem 24.8 hold under the key assumption $-\nabla f\left(x_{\infty}\right) \notin \mathrm{bd}(\partial \Phi(0))$. Since the boundary of the convex set $\partial \Phi(0)$ has an empty interior, it is reasonable to think that the circumstances leading to $-\nabla f\left(x_{\infty}\right) \in \mathrm{bd}(\partial \Phi(0))$ are "exceptional". More precisely, we conjecture that generically with respect to the initial data $\left(x_{0}, \dot{x}_{0}\right) \in \mathbb{R}^{n} \times \mathbb{R}^{n}$, the point $x_{\infty}=\lim _{t \rightarrow+\infty} x(t)$ satisfies the condition $-\nabla f\left(x_{\infty}\right) \notin \mathrm{bd}(\partial \Phi(0))$.

Let us now give a counterexample to finite time convergence when $-\nabla f\left(x_{\infty}\right) \in \operatorname{bd}(\partial \Phi(0))$. For that purpose, take $H=\mathbb{R}, \Phi:=||+.|.|^{2}$ (so that $\partial \Phi(0)=[-1,1]$ ) and $f:=|\cdot|^{2} / 2$. The differential inclusion $(S)$ then reduces to

$$
\ddot{x}(t)+\operatorname{Sgn}(\dot{x}(t))+2 \dot{x}(t)+x(t) \ni 0 .
$$

Let us choose as initial conditions $x(0)=2$ and $\dot{x}(0)=-1$. We let the reader check that the unique solution of $(S)$ is given by $x(t)=1+e^{-t}$, $t \geq 0$. The trajectory tends toward the value $x_{\infty}=1$, which satisfies $-f^{\prime}\left(x_{\infty}\right)=-1 \in \operatorname{bd}(\partial \Phi(0))$. However the convergence does not hold in a finite time.

\section{Proof of the existence result}

We prove the existence of a function $x:\left[0,+\infty\left[\rightarrow \mathbb{R}^{n}\right.\right.$ satisfying points (a), (b) and (c) of Theorem 24.1. For that purpose, let us define for any positive $\lambda$, the approximate equation

$$
\left(S_{\lambda}\right) \quad \ddot{x}_{\lambda}(t)+\nabla \Phi_{\lambda}\left(\dot{x}_{\lambda}(t)\right)+\nabla f\left(x_{\lambda}(t)\right)=0,
$$

where $\Phi_{\lambda}$ denotes the Moreau-Yosida approximate of $\Phi$. The general features relative to the Moreau-Yosida approximation can be found in Brezis (Brézis, 1972) or in Rockafellar-Wets (Rockafellar and Wets, 1998). Let us recall that, for any $\lambda>0, \Phi_{\lambda}$ is a $\mathcal{C}^{1}$ function from $\mathbb{R}^{n}$ into $\mathbb{R}$, whose gradient $\nabla \Phi_{\lambda}$ is Lipschitz continuous. Equation $\left(S_{\lambda}\right)$ falls into 
the field of ordinary differential equations. We let the reader check the following lemma.

Lemma 24.9 Assume $\left(\mathcal{H}_{f}-i, i i\right)$ and $\left(\mathcal{H}_{\Phi}-i, i i\right)$. Then, for every $\left(x_{0}, \dot{x}_{0}\right) \in \mathbb{R}^{n} \times \mathbb{R}^{n}$, there exists a unique maximal solution $x_{\lambda}:[0,+\infty[\rightarrow$ $\mathbb{R}^{n}$ of $\left(S_{\lambda}\right)$ satisfying $\left(x_{\lambda}(0), \dot{x}_{\lambda}(0)\right)=\left(x_{0}, \dot{x}_{0}\right)$. Moreover, setting $E_{\lambda}(t):=$ $\frac{1}{2}\left|\dot{x}_{\lambda}(t)\right|^{2}+f\left(x_{\lambda}(t)\right)$, we have for every $t \in \mathbb{R}_{+}$,

$$
\dot{E}_{\lambda}(t) \leq-\Phi_{\lambda}\left(\dot{x}_{\lambda}(t)\right)
$$

The proof of the local existence and uniqueness of $x_{\lambda}$ relies on the Cauchy-Lipschitz Theorem. Denoting by $\left[0, T_{\max }[\right.$ the maximal interval on which $x_{\lambda}$ is defined, we use an estimate of $\dot{x}_{\lambda}$ in $L^{\infty}\left(\left[0, T_{\max }\left[, \mathbb{R}^{n}\right)\right.\right.$ to prove that $T_{\max }=+\infty$. The decay property $(24.12)$ is trivial.

Coming back to the existence problem in Theorem 24.1, we are going to establish uniform estimates relying on the solutions of $\left(S_{\lambda}\right)$. Then arguing by compacity, we pass to the limit and exhibit a function $x$ which is proved to fulfil (a), (b) and (c).

Estimations. Since $\min _{\mathbb{R}^{n}} \Phi_{\lambda}=\min _{\mathbb{R}^{n}} \Phi=0$, we deduce from (24.12) that $\dot{E}_{\lambda} \leq 0$, i.e. $E_{\lambda}$ is a non increasing function. Therefore, we have

$$
\frac{1}{2}\left|\dot{x}_{\lambda}(t)\right|^{2}+f\left(x_{\lambda}(t)\right)=E_{\lambda}(t) \leq E_{\lambda}(0)=\frac{1}{2}\left|\dot{x}_{0}\right|^{2}+f\left(x_{0}\right)
$$

and since $f$ is bounded from below, we immediately infer that

$$
\left(\dot{x}_{\lambda}\right) \quad \text { is bounded in } L^{\infty}\left(\left[0,+\infty\left[, \mathbb{R}^{n}\right) .\right.\right.
$$

Let us now fix some $T>0$. From the formula $x_{\lambda}(t)=x_{0}+\int_{0}^{t} \dot{x}_{\lambda}(s) d s$, we deduce that

$$
\sup _{\substack{\lambda>0 \\ t \in[0, T]}}\left|x_{\lambda}(t)\right| \leq\left|x_{0}\right|+T \sup _{\lambda>0}\left\|\dot{x}_{\lambda}\right\|_{\infty}
$$

and hence

$$
\left(x_{\lambda}\right) \quad \text { is bounded in } L^{\infty}\left([0, T], \mathbb{R}^{n}\right) .
$$

From the last estimate (24.14), the boundedness of $\nabla f$ on the bounded sets implies that

$$
\left(\nabla f\left(x_{\lambda}\right)\right) \quad \text { is bounded in } L^{\infty}\left([0, T], \mathbb{R}^{n}\right) .
$$

On the other hand, denoting by $\partial \Phi^{0}(y)$ the element of minimal norm, it is well-known that $\left|\nabla \Phi_{\lambda}(y)\right| \leq\left|\partial \Phi^{0}(y)\right|$ for every $y \in \mathbb{R}^{n}$. As a consequence, we have $\left|\nabla \Phi_{\lambda}\left(\dot{x}_{\lambda}\right)\right| \leq\left|\partial \Phi^{0}\left(\dot{x}_{\lambda}\right)\right|$, which combined with estimate (24.13) and the boundedness of $\partial \Phi$ on bounded sets, gives

$$
\left(\nabla \Phi_{\lambda}\left(\dot{x}_{\lambda}\right)\right) \quad \text { is bounded in } L^{\infty}\left(\left[0,+\infty\left[, \mathbb{R}^{n}\right) .\right.\right.
$$


By taking into account (24.15) and (24.16), we deduce in view of $\left(S_{\lambda}\right)$ that

$$
\left(\ddot{x}_{\lambda}\right) \quad \text { is bounded in } L^{\infty}\left([0, T], \mathbb{R}^{n}\right) .
$$

Passing to the limit. From the inequality $\left|x_{\lambda}\left(t^{\prime}\right)-x_{\lambda}(t)\right| \leq$ $\left(\sup _{\lambda>0}\left\|\dot{x}_{\lambda}\right\|_{\infty}\right)\left|t^{\prime}-t\right|$, it ensues that $\left(x_{\lambda}\right)$ is an equicontinuous bounded sequence in $\mathcal{C}\left([0, T], \mathbb{R}^{n}\right)$ equipped with the supremum norm, and therefore Ascoli Theorem shows the existence of a cluster point $x \in \mathcal{C}\left([0, T], \mathbb{R}^{n}\right)$ to the sequence $\left(x_{\lambda}\right)$. Then, there exists a subsequence of $\left(x_{\lambda}\right)$, still denoted by $\left(x_{\lambda}\right)$ such that

$$
x_{\lambda} \rightarrow x \quad \text { in } \mathcal{C}\left([0, T], \mathbb{R}^{n}\right) .
$$

In view of estimate (24.17), the same argument applied to the sequence $\left(\dot{x}_{\lambda}\right)$ shows that there exist $u \in \mathbb{R}^{n}$ and a subsequence of $\left(\dot{x}_{\lambda}\right)$, still denoted by $\left(\dot{x}_{\lambda}\right)$ such that

$$
\dot{x}_{\lambda} \rightarrow u \quad \text { in } \mathcal{C}\left([0, T], \mathbb{R}^{n}\right) .
$$

Hence we have $\dot{x}_{\lambda} \rightarrow \dot{x}$ and $\dot{x}_{\lambda} \rightarrow u$ in the sense of distributions in ] $0, T$. Identifying both limits, we infer that $\dot{x} \in \mathcal{C}\left([0, T], \mathbb{R}^{n}\right)$, i.e. $x \in$ $\mathcal{C}^{1}\left([0, T], \mathbb{R}^{n}\right)$. From $(24.17)$, the sequence $\left(\ddot{x}_{\lambda}\right)$ is bounded in the space $L^{\infty}\left([0, T], \mathbb{R}^{n}\right)$, which can be identified with the topological dual of $L^{1}\left([0, T], \mathbb{R}^{n}\right)$. The Banach-Alaoglu Theorem then shows the existence of $v \in L^{\infty}\left([0, T], \mathbb{R}^{n}\right)$ and a subsequence of $\left(\ddot{x}_{\lambda}\right)$, still denoted by $\left(\ddot{x}_{\lambda}\right)$ such that

$$
\ddot{x}_{\lambda} \rightarrow v \quad \text { for the topology } \sigma\left(L^{\infty}\left([0, T], \mathbb{R}^{n}\right), L^{1}\left([0, T], \mathbb{R}^{n}\right)\right) .
$$

Hence, we have $\ddot{x}_{\lambda} \rightarrow \ddot{x}$ and $\ddot{x}_{\lambda} \rightarrow v$ in the sense of distributions in ] $0, T\left[\right.$. The identification of the limits shows that $\ddot{x} \in L^{\infty}\left([0, T], \mathbb{R}^{n}\right)$ or equivalently $x \in \mathcal{W}^{2, \infty}\left([0, T], \mathbb{R}^{n}\right)$.

Let us now prove that the map $x$ satisfies $(S)$ almost everywhere on $[0, T]$. Fix $\theta \geq 0$ in $\mathcal{C}_{c}(] 0, T[)$ (the set of continuous functions with compact support included in $] 0, T\left[\right.$ ). Since $\Phi_{\lambda}$ is convex, the following inequality holds for every $t \geq 0$ :

$$
\begin{aligned}
\forall \xi \in \mathbb{R}^{n}, \quad \Phi_{\lambda}(\xi) & \geq \Phi_{\lambda}\left(\dot{x}_{\lambda}(t)\right)+\left\langle\nabla \Phi_{\lambda}\left(\dot{x}_{\lambda}(t)\right), \xi-\dot{x}_{\lambda}(t)\right\rangle \\
& \geq \Phi_{\lambda}\left(\dot{x}_{\lambda}(t)\right)+\left\langle-\ddot{x}_{\lambda}(t)-\nabla f\left(x_{\lambda}(t)\right), \xi-\dot{x}_{\lambda}(t)\right\rangle .
\end{aligned}
$$

Multiplying both members by $\theta$ and integrating on $[0, T]$, we obtain:

$$
\begin{aligned}
\int_{0}^{T} \theta(t) \Phi_{\lambda}(\xi) d t \geq \int_{0}^{T} \theta(t) \Phi_{\lambda}\left(\dot{x}_{\lambda}(t)\right) d t+ \\
\\
\int_{0}^{T} \theta(t)\left\langle-\ddot{x}_{\lambda}(t)-\nabla f\left(x_{\lambda}(t)\right), \xi-\dot{x}_{\lambda}(t)\right\rangle d t .
\end{aligned}
$$


Fix some $\lambda_{0}>0$ and consider $\left.\lambda \in\right] 0, \lambda_{0}[$. From the monotonicity of the Moreau-Yosida approximation, we have $\Phi_{\lambda_{0}} \leq \Phi_{\lambda} \leq \Phi$ so that the last inequality implies

$$
\begin{aligned}
\int_{0}^{T} \theta(t) \Phi(\xi) d t \geq & \int_{0}^{T} \theta(t) \Phi_{\lambda_{0}}\left(\dot{x}_{\lambda}(t)\right) d t+ \\
& \int_{0}^{T} \theta(t)\left\langle-\ddot{x}_{\lambda}(t)-\nabla f\left(x_{\lambda}(t)\right), \xi-\dot{x}_{\lambda}(t)\right\rangle d t .
\end{aligned}
$$

Take now the limit when $\lambda \rightarrow 0$ in the previous inequality. Since $\Phi_{\lambda_{0}}$ is Lipschitz continuous on the bounded sets and since $\left(\dot{x}_{\lambda}\right)$ converges uniformly toward $\dot{x}$, we deduce that $\Phi_{\lambda_{0}}\left(\dot{x}_{\lambda}\right) \rightarrow \Phi_{\lambda_{0}}(\dot{x})$ uniformly in $\mathcal{C}\left([0, T], \mathbb{R}^{n}\right)$, whence

$$
\lim _{\lambda \rightarrow 0} \int_{0}^{T} \theta(t) \Phi_{\lambda_{0}}\left(\dot{x}_{\lambda}(t)\right) d t=\int_{0}^{T} \theta(t) \Phi_{\lambda_{0}}(\dot{x}(t)) d t .
$$

Similarly, the uniform convergence of $\left(x_{\lambda}\right)$ toward $x$ joined with the Lipschitz continuity of $\nabla f$ on the bounded sets shows that $\nabla f\left(x_{\lambda}\right) \rightarrow$ $\nabla f(x)$ uniformly in $\mathcal{C}\left([0, T], \mathbb{R}^{n}\right)$. In view of the weak convergence of $\left(\ddot{x}_{\lambda}\right)$ toward $\ddot{x}$ in the $\sigma\left(L^{\infty}, L^{1}\right)$ sense, this implies that

$$
\begin{aligned}
\lim _{\lambda \rightarrow 0} \int_{0}^{T} \theta(t)\left\langle-\ddot{x}_{\lambda}(t)-\right. & \left.\nabla f\left(x_{\lambda}(t)\right), \xi-\dot{x}_{\lambda}(t)\right\rangle d t= \\
& \int_{0}^{T} \theta(t)\langle-\ddot{x}(t)-\nabla f(x(t)), \xi-\dot{x}(t)\rangle d t .
\end{aligned}
$$

Let us take the limit when $\lambda \rightarrow 0$ in inequality (24.18) by taking into account (24.19) and (24.20):

$$
\begin{aligned}
\int_{0}^{T} \theta(t) \Phi(\xi) d t \geq & \int_{0}^{T} \theta(t) \Phi_{\lambda_{0}}(\dot{x}(t)) d t+ \\
& \int_{0}^{T} \theta(t)\langle-\ddot{x}(t)-\nabla f(x(t)), \xi-\dot{x}(t)\rangle d t .
\end{aligned}
$$

Taking the limit when $\lambda_{0} \rightarrow 0$ in the previous inequality and applying the Beppo-Levi Theorem, it is immediate that (24.21) holds with $\Phi$ in place of $\Phi_{\lambda_{0}}$. The latter being true for all $\theta \geq 0$ in $\mathcal{C}_{c}(] 0, T[)$, it follows that for every $\xi \in \mathbb{R}^{n}$,

$$
\Phi(\xi) \geq \Phi(\dot{x}(t))+\langle-\ddot{x}(t)-\nabla f(x(t)), \xi-\dot{x}(t)\rangle \quad \text { ae in }[0, T] .
$$

From the definition of the subdifferential, this is equivalent to:

$$
-\ddot{x}(t)-\nabla f(x(t)) \in \partial \Phi(\dot{x}(t)) \quad \text { ae in }[0, T],
$$


which means that inclusion $(S)$ is satisfied almost everywhere in $[0, T]$.

Let us now summarize our results. For every $T>0$, we have proved the existence of a function $x \in \mathcal{C}^{1}\left([0, T], \mathbb{R}^{n}\right) \cap \mathcal{W}^{2, \infty}\left([0, T], \mathbb{R}^{n}\right)$ such that $(S)$ is fulfilled for almost every $t \in[0, T]$. Since $\left(x_{\lambda}(0), \dot{x}_{\lambda}(0)\right)=\left(x_{0}, \dot{x}_{0}\right)$ for every $\lambda>0$, it is immediate that the limit function $x$ also satisfies $(x(0), \dot{x}(0))=\left(x_{0}, \dot{x}_{0}\right)$. To avoid confusion, we now denote by $x^{T}$ the previous function defined on $[0, T]$. For every $T^{\prime}>T$, we obtain in the same way a function $x^{T^{\prime}}$ defined on $\left[0, T^{\prime}\right]$. From the uniqueness result, it is clear that $x_{\mid[0, T]}^{T^{\prime}}=x^{T}$, so that we can define without any ambiguity a function $x:[0,+\infty[$ in the following manner:

$$
\forall t \in\left[0,+\infty\left[, \quad x(t)=x^{T}(t) \quad \text { as soon as } T \geq t .\right.\right.
$$

It is then immediate that such a function $x$ satisfies items (a), (b) and (c) of Theorem 24.1.

\section{References}

S. Adly, D. Goeleven, A stability theory for second order nonsmooth dynamical systems with application to friction problems, Journal de Mathématiques Pures et Appliquées, vol. 83, (2004), 17-51.

F. Alvarez, On the minimizing property of a second order dissipative system in Hilbert space, SIAM J. on Control and Optimization, vol. 38, 4 (2000), 1102-1119.

F. Alvarez, H. Attouch, J. Bolte, P. Redont, A second-order gradient-like dissipative dynamical system with Hessian-driven damping. Application to optimization and mechanics, J. Math. Pures Appl., vol. 81, 8 (2002), 747-779.

H. Amann, J. I. Díaz, A note on the dynamics of an oscillator in the presence of strong friction, Nonlinear Anal., vol. 55 (2003), 209-216.

A. Amassad, C. Fabre, Analysis of a viscoelastic unilateral contact problem involving the Coulomb friction law, J. Optim. Theory Appl., vol. 116, 3 (2003), 465-483.

H. Attouch, X. Goudou, P. Redont, The heavy ball with friction method. I The continuous dynamical system, Communications in Contemporary Math, vol. 2, 1 (2000), 1-34.

H. Attouch, P. Redont, The second-order in time continuous Newton method. Approximation, optimization and mathematical economics (Pointe--Pitre, 1999), 25-36, Physica, Heidelberg, 2001.

H. Brézis, Opérateurs maximaux monotones dans les espaces de Hilbert et équations d'évolution, Lecture Notes 5, North Holland, 1972.

M. Cadivel, D. Goeleven and M. Shillor, Study of a unilateral oscillator with friction, Math. Comput. Modelling, vol. 32, 2/3 (2000), 381-391. 
J. Conlisk, Why bounded rationality, Journal of Economic Literature, vol. 34 (1996), 669-700.

J. I. Díaz, A. Liñán, On the asymptotic behavior of a damped oscillator under a sublinear friction term, Rev. R. Acad. Cien. Serie A. Mat., vol. 95, 1 (2001), 155-160.

Y. Dumont, D. Goeleven, M. Rochdi and M. Shillor, Frictional contact of a nonlinear spring, Math. Comput. Modelling, vol. 31, 2/3 (2000), 83-97.

C. Eck, J. Jarusek, A survey on dynamic contact problems with Coulomb friction, Multifield problems, (2000), 254-261, Springer, Berlin.

W. Han, M. Sofonea, Quasistatic contact problems in viscoelasticity and viscoplasticity, AMS and International Press, 2002.

M. Jean, Unilateral contact with dry friction: time and space discrete variables formulation, Arch. Mech., vol. 40, 5/6 (1988), 677-691.

T. Kato, Accretive operators and nonlinear evolution equations in Banach spaces. Nonlinear functional analysis, Proc. Symp. Pure Math., vol. 18, 1 (1970), 138-161.

M. Mabrouk, Sur un principe variationnel pour un problème d'évolution hyperbolique non linéaire, working paper, Laboratoire de Mécanique Appliquée, Université de Franche-Comté, Besançon.

M. D. P. Monteiro Marques, Differential inclusions in nonsmooth mechanical problems, Progress in nonlinear differential equations and their applications, vol. 9, Birkhauser, 1993.

J. J. Moreau, Evolution problem associated with a moving convex set in a Hilbert space, J. Diff. Equ., (1977), 347-374.

J. J. Moreau, Standard inelastic shocks and the dynamics of unilateral constraints. In: Unilateral Problems in Structural Analysis (ed. by G. Del Piero and F. Maceri), CISM Courses and Lectures, vol. 288, Springer Verlag, Wien, New York 1985, 173-221.

B.T. Polyack, Some methods of speeding up the convergence of iterative methods, Z. Vylist Math. Fiz., vol. 4, (1964), 1-17.

R.T. Rockafellar and R. Wets, Variational analysis, Springer, Berlin, 1998.

A. Rubinstein, Modeling bounded rationality, (1998), MIT Press.

D. A. Russell, The piano hammer as a nonlinear spring, publications of the Science and Mathematics Department, GMI Engineering \& Management Institute, Kettering University, 1997.

M. Schatzman, A class of nonlinear differential equations of second order in time, Nonlinear Analysis, vol. 2, (1978), 355-373.

J. Sobel, Economists' models of learning, Journal of Economics Theory, vol. 94, (2000), 241-261. 